\title{
ESTIMATES UP TO THE BOUNDARY OF A WEAK SOLUTION TO THE NAVIER-STOKES EQUATION IN A CUBE IN DEPENDENCE ON EIGENVALUES OF THE RATE OF DEFORMATION TENSOR
}

\author{
JIŘÍ NEUSTUPA \\ Department of Mathematics, Faculty of Mechanical Engineering, Czech Technical University \\ Karlovo nám. 13, 12135 Praha 2, Czech Republic \\ E-mail: neustupa@marian.fsik.cvut.cz \\ PATRICK PENEL \\ Université du Sud-Toulon-Var, Mathématique, BP 20132, 83957 La Garde, France \\ E-mail: penel@univ-tln.fr
}

\begin{abstract}
We formulate sufficient conditions for regularity up to the boundary of a weak solution $\boldsymbol{v}$ in a subdomain $\Omega \times\left(t_{1}, t_{2}\right)$ of the time-space cylinder $\Omega \times(0, T)$ by means of requirements on one of the eigenvalues of the rate of deformation tensor. We assume that $\Omega$ is a cube.
\end{abstract}

1. Introduction and auxiliary lemmas. We assume that $\Omega$ is a cube $(-\pi, \pi)^{3}$ in this paper.

We have already shown in our two previous papers [9] and [10] that the interior regularity of a so called suitable weak solution $\boldsymbol{v}$ to the Navier-Stokes equation can be guaranteed by certain assumptions on only one of the eigenvalues of the rate of deformation tensor (= the symmetrized gradient of velocity). The main reason why we studied only the interior regularity in [9] and [10] was that we assumed that $\boldsymbol{v}$ satisfied a Dirichlet boundary condition on $\partial \Omega$ and we were not able to perform all necessary integrations by parts and to derive a fundamental estimate of $\nabla \boldsymbol{v}$ on the whole domain $\Omega$.

In this paper, instead of the usual Dirichlet boundary condition, we assume that the considered weak solution $\boldsymbol{v}$ satisfies so called "vorticity boundary conditions" (4) introduced in the paper [1] by H. Bellout, J. Neustupa and P. Penel. Using a special and fine treatment of the boundary integrals, we show that a similar procedure as in [10]

2000 Mathematics Subject Classification: 35Q30, 76D05.

Key words and phrases: Navier-Stokes equations.

The paper is in final form and no version of it will be published elsewhere. 
enables us to obtain a desired estimate of $\nabla \boldsymbol{v}$. Thus, we derive a result on the regularity which is valid up to the boundary of $\Omega$ in Section 3 .

In Section 2, as an auxiliary result, we prove a theorem on local in time existence of a strong solution-see Theorem 5. A similar theorem is well known in the case of the Dirichlet boundary condition. The theorem, valid in the case of the boundary conditions (4), is also cited without proof in our paper [1]. However, in our opinion, the situation when the boundary of $\Omega$ is not smooth requires a special attention. Theorem 5 further enables to derive an important information on the structure of a weak solution that satisfies a so called strong energy inequality — see Theorem 6.

The advantage of $\Omega$ being the cube is that we know the explicit form of eigenfunctions of certain operator $A$ (coinciding with curl) - see Lemma 2. It enables to prove Lemma 3 and Lemma 4 whose conclusions play an important role in the paper. The other advantage is that we can show that the surface integral in (39) equals zero-see Section 3. We believe that the main result of the paper (Theorem 7 ) is also valid if $\Omega$ is e.g. a convex polyhedron, at least we are also able to show formally that the surface integral in (39) is zero in this case. However we are actually not able to verify whether Lemmas 3 and 4 are also valid on the convex polyhedron.

Let $T$ be a positive number and $Q_{T}=\Omega \times(0, T)$. We deal with the Navier-Stokes initial-boundary value problem which is defined by the equations

$$
\begin{array}{rlrl}
\frac{\partial \boldsymbol{v}}{\partial t}+(\boldsymbol{v} \cdot \nabla) \boldsymbol{v} & =-\nabla p+\nu \Delta \boldsymbol{v} & \text { in } Q_{T}, \\
\operatorname{div} \boldsymbol{v} & =0 \quad \text { in } Q_{T},
\end{array}
$$

by the initial condition

$$
\left.\boldsymbol{v}\right|_{t=0}=\boldsymbol{v}_{0}
$$

and by the vorticity boundary conditions

$$
\operatorname{curl}^{k} \boldsymbol{v} \cdot \boldsymbol{n}=0 \quad(k=0,1,2) \quad \text { on } \partial \Omega \times(0, T) .
$$

$\boldsymbol{v}=\left(v_{1}, v_{2}, v_{3}\right)$ denotes the velocity, $p$ denotes the pressure and $\nu>0$ is the viscosity coefficient. Throughout the paper, we shall use the usual notation:

- $\boldsymbol{n}=\left(n_{1}, n_{2}, n_{3}\right)$ is the outer normal vector on $\partial \Omega$.

- $\|\cdot\|_{r}$, respectively $\|\cdot\|_{m, r}$, is the norm of a scalar- or vector- or tensor-valued function with its components in $L^{r}(\Omega)$, respectively in $W^{m, r}(\Omega)$.

- $L_{\sigma}^{2}(\Omega)^{3}$ is the closure of $\left\{\boldsymbol{u} \in C_{0}^{\infty}(\Omega)^{3}\right.$; $\operatorname{div} \boldsymbol{u}=0$ in $\left.\Omega\right\}$ in $L^{2}(\Omega)^{3} . L_{\sigma}^{2}(\Omega)^{3}$ coincides with the set $\left\{\boldsymbol{u} \in L^{2}(\Omega)^{3} ; \operatorname{div} \boldsymbol{u}=0\right.$ in $\Omega$ in the sense of distributions and $\left.(\boldsymbol{u} \cdot \boldsymbol{n})\right|_{\partial \Omega}=0$ in the sense of traces\}.

- $(., .)_{2}$ denotes the scalar product in $L^{2}(\Omega)^{3}$ and in $L_{\sigma}^{2}(\Omega)^{3}$.

- $P_{\sigma}$ is the orthogonal projection of $L^{2}(\Omega)^{3}$ onto $L_{\sigma}^{2}(\Omega)^{3}$.

The notion of a weak solution to the problem (1)-(3) with the Dirichlet boundary condition

$$
\boldsymbol{v}=\mathbf{0} \quad \text { on } \partial \Omega \times(0, T) .
$$

is well known. The existence of a weak solution to the problem (1)-(3), (5) is a classical 
result of J. Leray [7] and E. Hopf [5]. (See also O. A. Ladyzhenskaya [6], R. Temam [11], M. Feistauer [3] and G. P. Galdi [4].) The weak solution $\boldsymbol{v}$, unlike others, belongs to $L^{2}\left(0, T ; W_{0}^{1,2}(\Omega)^{3}\right) \cap L^{\infty}\left(0, T ; L_{\sigma}^{2}(\Omega)^{3}\right)$. If $\boldsymbol{v}$ is a so called "suitable weak solution" then the set of singular points of $\boldsymbol{v}$ in $Q_{T}$ has the 1-dimensional Hausdorff measure equal to zero (see L. Caffarelli, R. Kohn and L. Nirenberg [2]).

We denote by $\sigma$ the symmetric part of the gradient of $\boldsymbol{v}$, i.e. $\sigma=\frac{1}{2}\left[\nabla \boldsymbol{v}+(\nabla \boldsymbol{v})^{T}\right] . \sigma$ is also called "the rate of deformation tensor". Let us recall our result from [9] and [10] where we were interested in the interior regularity of a suitable weak solution $\boldsymbol{v}$.

THEOREM 1. Suppose that $D$ is a subdomain of $Q_{T}$ and $\boldsymbol{v}$ is a suitable weak solution to the initial-boundary value problem (1)-(3), (5) in $Q_{T}$. Let $\zeta_{1} \leq \zeta_{2} \leq \zeta_{3}$ be the eigenvalues of the rate of deformation tensor $\sigma$. Suppose that

(i) one of the functions $\zeta_{1},\left(\zeta_{2}\right)_{+}, \zeta_{3}$ belongs to $L_{\text {loc }}^{r, s}(D)$ for some real numbers $r$, s such that $1 \leq r \leq+\infty, \frac{3}{2}<s \leq+\infty$ and $2 / r+3 / s \leq 2$.

$\left(\left(\zeta_{2}\right)_{+}\right.$denotes the positive part of $\left.\zeta_{2} \cdot\right)$ Then the solution $\boldsymbol{v}$ is regular in $D$.

$\sigma$, as a symmetric $3 \times 3$ tensor, has at most three real eigenvalues. The eigenvalues are functions of $\boldsymbol{x}$ and $t$. However, they can be denoted in a.a. points $(\boldsymbol{x}, t) \in D$ so that the least is $\zeta_{1}(\boldsymbol{x}, t)$, the middle is $\zeta_{2}(\boldsymbol{x}, t)$ and the greatest is $\zeta_{3}(\boldsymbol{x}, t)$. In fact, the theorem can also be reformulated in such a way that instead of assumption (i), we can define a function which coincides at a.a. points $(\boldsymbol{x}, t)$ with one of the eigenvalues, no matter with which of them, and make an analogous assumption on this function.

Let us note that even if the domain $D$ has for example the form $\Omega \times\left(t_{1}, t_{2}\right)$ (where $\left.0 \leq t_{1}<t_{2} \leq T\right)$ then the statement of Theorem 1 only says that $\boldsymbol{v}$ has no singular points in $D$, but it does not provide a new information on $\boldsymbol{v}$ as an element of a function space on $\Omega \times\left(t_{1}, t_{2}\right)$. This is exactly the point which will be improved in Section 3 , however $\boldsymbol{v}$ will be supposed to satisfy the boundary conditions (4) instead of (5).

The Navier-Stokes equation with the boundary conditions (4) was treated in [1]. Let us recall some notation and facts from [1]:

- $D^{1}=\left\{\boldsymbol{u} \in W^{1,2}(\Omega)^{3} \cap L_{\sigma}^{2}(\Omega)^{3} ;\left.(\operatorname{curl} \boldsymbol{u} \cdot \boldsymbol{n})\right|_{\partial \Omega}=0\right.$ in the sense of traces $\}$

- $A=\left.\operatorname{curl}\right|_{D^{1}}$

- The equation $A \boldsymbol{u}=\boldsymbol{f}$ (for $\boldsymbol{f} \in L_{\sigma}^{2}(\Omega)^{3}$ ) has a unique solution $\boldsymbol{u} \in D^{1}$ such that

$$
\|\boldsymbol{u}\|_{1,2} \leq c_{1}\|\boldsymbol{f}\|_{2}
$$

(where constant $c_{1}$ is independent of $\boldsymbol{f}$ ).

- There exist constants $c_{2}, c_{3}>0$ such that

$$
c_{2}\|A \boldsymbol{w}\|_{2} \leq\|\boldsymbol{w}\|_{1,2} \leq c_{3}\|A \boldsymbol{w}\|_{2} \text { for all } \boldsymbol{w} \in D^{1} .
$$

- $D^{1}$ can be described in this way:

(8) $D^{1}=\left\{\boldsymbol{u}=\boldsymbol{u}_{0}+\nabla \varphi ; \boldsymbol{u}_{0} \in W_{0}^{1,2}(\Omega)^{3}, \Delta \varphi=-\nabla \cdot \boldsymbol{u}_{0}\right.$ in $\Omega$ and $\left.\partial \varphi /\left.\partial \boldsymbol{n}\right|_{\partial \Omega}=0\right\}$.

- $A$ is a selfadjoint operator in $L_{\sigma}^{2}(\Omega)^{3}$ and the resolvent operator $(\lambda I-A)^{-1}$ is compact in $L_{\sigma}^{2}(\Omega)^{3}$ for all $\lambda$ from the resolvent set of $A$.

- The spectrum $\sigma(A)$ consists of countably many isolated real eigenvalues $\left\{\lambda_{i} ; i \in\right.$ $\left.\mathbf{Z}^{*}\right\} \quad\left(\mathbf{Z}^{*}=\mathbf{Z}-\{0\}\right)$. Each of the eigenvalues has the same finite algebraic and geometric 
multiplicity. The eigenvalues can be ordered so that $\lambda_{i}<0$ if $i<0, \lambda_{i}>0$ if $i>0$ and $\lambda_{i} \leq \lambda_{j}$ if $i<j$. The corresponding eigenfunctions $\boldsymbol{e}^{i}$ can be chosen so that they form a complete orthonormal system in $L_{\sigma}^{2}(\Omega)^{3}$.

We shall denote by $D^{2}$ the domain of $A^{2}$, i.e. the space $D\left(A^{2}\right) . \mathbf{Z}_{n}^{*}$ will denote the set of the integers $\{-n, \ldots,-1,1, \ldots, n\}, \boldsymbol{V}^{n}$ will be the space spanned by the eigenfunctions $\boldsymbol{e}^{i}$ for $i \in \mathbf{Z}_{n}^{*}$ and $\Pi_{n}$ will denote the orthogonal projection of $L_{\sigma}^{2}(\Omega)^{3}$ onto $\boldsymbol{V}^{n}$.

Lemma 2. The eigenfunctions $\boldsymbol{e}^{i}\left(i \in \mathbf{Z}^{*}\right)$ of operator $A$ satisfy

$$
\left(\nabla^{2} \boldsymbol{e}^{i}, \nabla^{2} \boldsymbol{e}^{j}\right)_{2}=\lambda_{i}^{2} \lambda_{j}^{2}\left(\boldsymbol{e}^{i}, \boldsymbol{e}^{j}\right)_{2}=\lambda_{i}^{2} \lambda_{j}^{2} \delta_{i j}
$$

where $\delta_{i j}$ is Kronecker's symbol.

Proof. One can deduce from the equations $A \boldsymbol{e}^{i}=\lambda_{i} \boldsymbol{e}^{i}$ and $A \boldsymbol{e}^{j}=\lambda_{j} \boldsymbol{e}^{j}$ that the eigenfunctions $\boldsymbol{e}^{i}$ and $\boldsymbol{e}^{j}$ must have the form

$$
\boldsymbol{e}^{i}(\boldsymbol{x})=\sum_{|k|=\lambda_{i}^{2}} \boldsymbol{c}_{k}^{i} \exp (\mathrm{i} \boldsymbol{x} \cdot \boldsymbol{k}), \quad \boldsymbol{e}^{j}(\boldsymbol{x})=\sum_{|l|=\lambda_{j}^{2}} \boldsymbol{c}_{l}^{j} \exp (\mathrm{i} \boldsymbol{x} \cdot \boldsymbol{k})
$$

where $\boldsymbol{k}=\left(k_{1}, k_{2}, k_{3}\right) \in \mathbf{Z}^{3}, \boldsymbol{l}=\left(l_{1}, l_{2}, l_{3}\right) \in \mathbf{Z}^{3}, \boldsymbol{c}_{k}^{i} \in \mathbb{R}^{3}$ and $\boldsymbol{c}_{l}^{j} \in \mathbb{R}^{3}$. (See [1], example 3.1.) Thus, $\lambda_{i}$ and $\lambda_{j}$ must also necessarily have the special forms $\lambda_{i}= \pm \sqrt{k_{1}^{2}+k_{2}^{2}+k_{3}^{2}}$ and $\lambda_{j}= \pm \sqrt{l_{1}^{2}+l_{2}^{2}+l_{3}^{2}}$. The coefficients $\boldsymbol{c}_{k}^{i}$ must be chosen so that function $\boldsymbol{e}^{i}$ expressed by (10) satisfies the equation $\operatorname{curl} \boldsymbol{e}^{i}=\lambda_{i} \boldsymbol{e}^{i}$ and the boundary condition $\boldsymbol{e}^{i} \cdot \boldsymbol{n}=0$ a.e. on $\partial \Omega$. The same holds on the coefficients $\boldsymbol{c}_{l}^{j}$. As an example, the exact form of an eigenfunction corresponding to $\lambda_{i}=\sqrt{1^{2}+2^{2}+3^{2}}=\sqrt{14}$ is shown in [1]. We have:

$$
\begin{aligned}
& \left(\nabla^{2} \boldsymbol{e}^{i}, \nabla^{2} \boldsymbol{e}^{j}\right)_{2}=\int_{\Omega} \sum_{|k|^{2}=\lambda_{i}^{2}} \boldsymbol{c}_{k}^{i}\left(-k_{r} k_{s}\right) \exp (\mathrm{i} \boldsymbol{x} \cdot \boldsymbol{k}) \sum_{|l|^{2}=\lambda_{j}^{2}} \overline{\boldsymbol{c}_{l}^{j}}\left(-l_{r} l_{s}\right) \exp (-\mathrm{i} \boldsymbol{x} \cdot \boldsymbol{l}) \mathrm{d} \boldsymbol{x} \\
& =\sum_{|k|^{2}=\lambda_{i}^{2}} \sum_{|l|^{2}=\lambda_{j}^{2}} \boldsymbol{c}_{k}^{i} \overline{\boldsymbol{c}_{l}^{j}} k_{r} l_{r} k_{s} l_{s} \int_{\Omega} \exp [\mathrm{i} \boldsymbol{x} \cdot(\boldsymbol{k}-\boldsymbol{l})] \mathrm{d} \boldsymbol{x}=\sum_{|k|^{2}=\lambda_{i}^{2}=\lambda_{j}^{2}} \boldsymbol{c}_{k}^{i} \overline{\boldsymbol{c}_{k}^{j}} k_{r} k_{r} k_{s} k_{s} \\
& =|\boldsymbol{k}|^{4} \sum_{|k|^{2}=\lambda_{i}^{2}=\lambda_{j}^{2}} \boldsymbol{c}_{k}^{i} \overline{\boldsymbol{c}_{k}^{j}}=\lambda_{i}^{2} \lambda_{j}^{2}\left(\boldsymbol{e}^{i}, \boldsymbol{e}^{j}\right)_{2}=\lambda_{i}^{2} \lambda_{j}^{2} \delta_{i j} .
\end{aligned}
$$

(The integral on $\Omega$ is different from zero only if $\boldsymbol{k}=\boldsymbol{l}$.) This completes the proof.

LEMMA 3. If $\boldsymbol{f} \in L_{\sigma}^{2}(\Omega)^{3}$ then the equation $A^{2} \boldsymbol{u}=\boldsymbol{f}$ has a unique solution $\boldsymbol{u} \in W^{2,2}(\Omega)^{3}$. The solution satisfies the estimate

$$
\|\boldsymbol{u}\|_{2,2}^{2} \leq\|\boldsymbol{f}\|_{2}^{2}+\|A \boldsymbol{u}\|_{2}^{2}+\|\boldsymbol{u}\|_{2}^{2}
$$

Proof. The existence of a unique solution $\boldsymbol{u} \in D\left(A^{2}\right)$ can be obtained by solving successively the two problems $A \boldsymbol{w}=\boldsymbol{f}$ and $A \boldsymbol{u}=\boldsymbol{w}$. We need to show that $\boldsymbol{u} \in W^{2,2}(\Omega)^{3}$ and $\boldsymbol{u}$ satisfies (11). Put

$$
\boldsymbol{u}^{n}=\Pi_{n} \boldsymbol{u}=\sum_{i \in Z_{n}^{*}}\left(\boldsymbol{u}, \boldsymbol{e}^{i}\right)_{2} \boldsymbol{e}^{i}
$$

Obviously, $\boldsymbol{u}^{n} \rightarrow \boldsymbol{u}$ as $n \rightarrow+\infty$ in the norm $\left\{\|\cdot\|_{2}^{2}+\|A \cdot\|_{2}^{2}+\left\|A^{2} \cdot\right\|_{2}^{2}\right\}^{1 / 2}$ which is 
equivalent with $\left\{\|\cdot\|_{1,2}^{2}+\|\operatorname{curl} \cdot\|_{1,2}^{2}\right\}^{1 / 2}$. Due to Lemma 2, we have:

$$
\left\|\nabla^{2} \boldsymbol{u}^{n}\right\|_{2}^{2}=\left\|A^{2} \boldsymbol{u}^{n}\right\|_{2}^{2}=\sum_{i \in Z_{n}^{*}}\left(\boldsymbol{u}, \boldsymbol{e}^{i}\right)_{2}^{2} \lambda_{i}^{4} \leq \sum_{i \in Z^{*}}\left(\boldsymbol{u}, \boldsymbol{e}^{i}\right)_{2}^{2} \lambda_{i}^{4}=\left\|A^{2} \boldsymbol{u}\right\|_{2}^{2} .
$$

We can also derive a similar estimate of $\left\|\nabla \boldsymbol{u}^{n}\right\|_{2}$. Hence the sequence $\left\{\boldsymbol{u}^{n}\right\}$ is bounded in $W^{2,2}(\Omega)^{3}$ and the limit $\boldsymbol{u}$ belongs to $W^{2,2}(\Omega)^{3}$. Moreover, it satisfies

$\|\boldsymbol{u}\|_{2,2}^{2}=\left\|\nabla^{2} \boldsymbol{u}\right\|_{2}^{2}+\|\nabla \boldsymbol{u}\|_{2}^{2}+\|\boldsymbol{u}\|_{2}^{2} \leq\left\|A^{2} \boldsymbol{u}\right\|_{2}^{2}+\|A \boldsymbol{u}\|_{2}^{2}+\|\boldsymbol{u}\|_{2}^{2}=\|\boldsymbol{f}\|_{2}^{2}+\|A \boldsymbol{u}\|_{2}^{2}+\|\boldsymbol{u}\|_{2}^{2}$.

This completes the proof.

We can deduce from Lemma 3 that $D^{2}$ is a subspace of $W^{2,2}(\Omega)^{3}$ and the norms $\|\cdot\|_{2,2}$ and $\left\{\|\cdot\|_{2}^{2}+\|A \cdot\|_{2}^{2}+\left\|A^{2} \cdot\right\|_{2}^{2}\right\}^{1 / 2}$ are equivalent in $D^{2}$.

LEMma 4. If $\boldsymbol{f} \in L_{\sigma}^{2}(\Omega)^{3}$ then the equation $A^{3} \boldsymbol{u}=\boldsymbol{f}$ has a unique solution $\boldsymbol{u} \in W^{3,2}(\Omega)^{3}$. The solution satisfies the estimate

$$
\|\boldsymbol{u}\|_{3,2}^{2} \leq\|\boldsymbol{f}\|_{2}^{2}+\left\|A^{2} \boldsymbol{u}\right\|_{2}^{2}+\|A \boldsymbol{u}\|_{2}^{2}+\|\boldsymbol{u}\|_{2}^{2} .
$$

We do not give the proof of Lemma 4 because it could be done similarly as the proof of Lemma 3.

Using the operator $A$, we can rewrite equation (1) and boundary conditions (4):

$$
\begin{aligned}
\frac{\partial \boldsymbol{v}}{\partial t}+A \boldsymbol{v} \times \boldsymbol{v} & =-\nabla\left(p+\frac{1}{2}|\boldsymbol{v}|^{2}\right)-\nu A^{2} \boldsymbol{v} \quad \text { in } Q_{T}, \\
A^{k} \boldsymbol{v} \cdot \boldsymbol{n} & =0 \quad(k=0,1,2) \quad \text { on } \partial \Omega \times(0, T) .
\end{aligned}
$$

As we have already mentioned, the existence of a weak solution to the problem (1)-(3), (5) is well known. Considering the boundary conditions (14) instead of (5) and assuming that $\boldsymbol{v}_{0} \in L_{\sigma}^{2}(\Omega)^{3}$, we can follow the proof described in [6], [11], [3] and [4] and recover the existence of a weak solution $\boldsymbol{v}$ of the problem (13), (2), (3) and (14) as a function from $L^{2}\left(0, T ; D^{1}\right) \cap L^{\infty}\left(0, T ; L_{\sigma}^{2}(\Omega)^{3}\right)$, satisfying the integral relation

$$
\begin{aligned}
\int_{0}^{T} & \int_{\Omega} \boldsymbol{v} \cdot \boldsymbol{\phi}(\boldsymbol{x}) \dot{\theta}(t) \mathrm{d} \boldsymbol{x} \mathrm{d} t-\int_{0}^{T} \int_{\Omega}[(A \boldsymbol{v} \times \boldsymbol{v}) \cdot \boldsymbol{\phi}(\boldsymbol{x})+\nu A \boldsymbol{v} \cdot A \phi(\boldsymbol{x})] \theta(t) \mathrm{d} \boldsymbol{x} \mathrm{d} t \\
& +\int_{\Omega} \boldsymbol{v}_{0} \cdot \boldsymbol{\phi}(\boldsymbol{x}) \theta(0) \mathrm{d} \boldsymbol{x}=0
\end{aligned}
$$

for all $\phi \in D^{1}$ and all $\theta \in C^{\infty}([0, T])$ such that $\theta(T)=0$. (See also [1] for further comments.)

$\boldsymbol{v}$ satisfies the first two conditions in (14) (corresponding to $k=0,1)$ for a.a. $t \in(0, T)$, as a function from $D^{1}$. A natural question is how we can understand the third condition in (14) which says that $A^{2} \boldsymbol{v} \cdot \boldsymbol{n}=0$ on $\partial \Omega \times(0, T)$. We can offer two explanations:

- The definition of the weak solution already contains the third boundary condition in itself in such a sense that if a weak solution $\boldsymbol{v}$ is smooth enough then it must already satisfy the condition $A^{2} \boldsymbol{v} \cdot \boldsymbol{n}=0$ on $\partial \Omega \times(0, T)$. This can be shown by the same procedure as in [1], the proof of Lemma 4.1.

○ We shall show in Section 2 that $A^{2} \boldsymbol{v}(., t) \in W^{1,2}(\Omega)^{3}$ for a.a. $t \in(0, T)$. Thus, $\boldsymbol{v}$ satisfies the third condition in (14) in the sense of traces in a.a. times $t$ in $(0, T)$. 


\section{The local in time existence of a strong solution and the structure theorem}

THEOREM 5. Let $j$ be either 1 or 2 . If $\boldsymbol{v}_{0} \in D^{j}$ then there exists $T_{j}^{*}>0$ and a function $\boldsymbol{v} \in C\left(0, T_{j}^{*} ; D^{j}\right)$ such that $A^{j+1} \boldsymbol{v} \in L^{2}\left(0, T_{j}^{*} ; L_{\sigma}^{2}(\Omega)^{3}\right), \partial A^{j-1} \boldsymbol{v} / \partial t \in L^{2}\left(0, T_{j}^{*} ; L_{\sigma}^{2}(\Omega)^{3}\right)$ and $\boldsymbol{v}$ satisfies (15) with $T=T_{j}^{*}$. Moreover, $T_{j}^{*}$ is a decreasing function of $\left\|A^{j} \boldsymbol{v}_{0}\right\|_{2}$.

Proof. Suppose that $j=1$ at first. Let $n \in \mathbb{N}$. Let us construct approximations of the solution $\boldsymbol{v}$ in the form

$$
\boldsymbol{v}^{n}(\boldsymbol{x}, t)=\sum_{i \in Z_{n}^{*}} a_{i}(t) \boldsymbol{e}^{i}(\boldsymbol{x})
$$

so that

$$
\begin{gathered}
\forall i \in \mathbf{Z}_{n}^{*}: \quad \frac{\mathrm{d}}{\mathrm{d} t} \int_{\Omega} \boldsymbol{v}^{n} \cdot \boldsymbol{e}^{i} \mathrm{~d} \boldsymbol{x}+\int_{\Omega}\left(A \boldsymbol{v}^{n} \times \boldsymbol{v}^{n}\right) \cdot \boldsymbol{e}^{i} \mathrm{~d} \boldsymbol{x}+\nu \int_{\Omega} A \boldsymbol{v}^{n} \cdot A \boldsymbol{e}^{i} \mathrm{~d} \boldsymbol{x}=0, \\
\boldsymbol{v}^{n}(., 0)=\Pi_{n} \boldsymbol{v}_{0} .
\end{gathered}
$$

(17), (18) is equivalent with the initial-value problem for the system of ordinary differential equations:

$$
\begin{gathered}
\frac{\mathrm{d}}{\mathrm{d} t} a_{i}(t)+\sum_{j, k \in Z_{n}^{*}} \lambda_{j} a_{j}(t) a_{k}(t) b_{i j k}+\nu \lambda_{i}^{2} a_{i}(t)=0 ; \quad i \in \mathbf{Z}_{n}^{*}, \\
a_{i}(0)=\left(\boldsymbol{v}_{0}, \boldsymbol{e}^{i}\right)_{2} ; \quad i \in \mathbf{Z}_{n}^{*},
\end{gathered}
$$

where

$$
b_{i j k}=\int_{\Omega} \boldsymbol{e}^{i} \cdot\left(\boldsymbol{e}^{j} \times \boldsymbol{e}^{k}\right) \mathrm{d} \boldsymbol{x} .
$$

The problem $(19),(20)$ has a solution in certain time interval $\left(0, T^{*}\right)$. In order to derive a lower estimate of $T^{*}$, we multiply the $i$-th equation in (19) by $\lambda_{i}^{2} a_{i}$ and sum over $i \in \mathbf{Z}_{n}^{*}$. We obtain

$$
\frac{\mathrm{d}}{\mathrm{d} t} \frac{1}{2} \sum_{i \in Z_{n}^{*}} \lambda_{i}^{2} a_{i}^{2}+\sum_{i, j, k \in Z_{n}^{*}} \lambda_{i}^{2} \lambda_{j} a_{i} a_{j} a_{k} b_{i j k}+\nu \sum_{i \in Z_{n}^{*}} \lambda_{i}^{4} a_{i}^{2}=0 .
$$

This is equivalent with

$$
\frac{\mathrm{d}}{\mathrm{d} t} \frac{1}{2} \int_{\Omega}\left|A \boldsymbol{v}^{n}\right|^{2} \mathrm{~d} \boldsymbol{x}+\int_{\Omega}\left(A \boldsymbol{v}^{n} \times \boldsymbol{v}^{n}\right) \cdot A^{2} \boldsymbol{v}^{n} \mathrm{~d} \boldsymbol{x}+\nu \int_{\Omega}\left|A^{2} \boldsymbol{v}^{n}\right|^{2} \mathrm{~d} \boldsymbol{x}=0 .
$$

The second term on the left-hand side can be estimated as follows:

$$
\left|\int_{\Omega}\left(A \boldsymbol{v}^{n} \times \boldsymbol{v}^{n}\right) \cdot A^{2} \boldsymbol{v}^{n} \mathrm{~d} \boldsymbol{x}\right| \leq \frac{\nu}{4} \int_{\Omega}\left|A^{2} \boldsymbol{v}^{n}\right|^{2} \mathrm{~d} \boldsymbol{x}+\frac{1}{\nu} \int_{\Omega}\left|A \boldsymbol{v}^{n}\right|^{2}\left|\boldsymbol{v}^{n}\right|^{2} \mathrm{~d} \boldsymbol{x}
$$

where

$$
\begin{aligned}
& \frac{1}{\nu} \int_{\Omega}\left|A \boldsymbol{v}^{n}\right|^{2}\left|\boldsymbol{v}^{n}\right|^{2} \mathrm{~d} \boldsymbol{x} \leq \frac{1}{\nu}\left(\int_{\Omega}\left|A \boldsymbol{v}^{n}\right|^{6} \mathrm{~d} \boldsymbol{x}\right)^{1 / 6}\left(\int_{\Omega}\left|A \boldsymbol{v}^{n}\right|^{2} \mathrm{~d} \boldsymbol{x}\right)^{1 / 2}\left(\int_{\Omega}\left|\boldsymbol{v}^{n}\right|^{6} \mathrm{~d} \boldsymbol{x}\right)^{1 / 3} \\
& \leq \delta \nu\left(\int_{\Omega}\left|A \boldsymbol{v}^{n}\right|^{6} \mathrm{~d} \boldsymbol{x}\right)^{1 / 3}+\frac{c_{4}}{\nu^{2} \delta}\left\|\boldsymbol{v}^{n}\right\|_{1,2}^{4}\left\|A \boldsymbol{v}^{n}\right\|_{2}^{2} \\
& \leq \delta \nu c_{4}\left\|A \boldsymbol{v}^{n}\right\|_{1,2}^{2}+\frac{c_{4} c_{3}}{\nu^{2} \delta}\left\|A \boldsymbol{v}^{n}\right\|_{2}^{6} \leq \delta \nu c_{4}\left\|A^{2} \boldsymbol{v}^{n}\right\|_{2}^{2}+\frac{c_{4} c_{3}}{\nu^{2} \delta}\left\|A \boldsymbol{v}^{n}\right\|_{2}^{6} .
\end{aligned}
$$


(We have estimated $\left\|\boldsymbol{v}^{n}\right\|_{1,2}$ and $\left\|A \boldsymbol{v}^{n}\right\|_{1,2}$ by means of (7).) Choosing $\delta>0$ so small that $\delta c_{4}<\frac{1}{4}$ and using the above estimates in (22), we can obtain:

$$
\frac{\mathrm{d}}{\mathrm{d} t} \frac{1}{2}\left\|A \boldsymbol{v}^{n}\right\|_{2}^{2}+\frac{\nu}{2}\left\|A^{2} \boldsymbol{v}^{n}\right\|_{2}^{2} \leq \frac{c_{4} c_{3}}{\nu^{2} \delta}\left\|A \boldsymbol{v}^{n}\right\|_{2}^{6}
$$

Integrating this inequality, we can show that

$$
\left\|A \boldsymbol{v}^{n}(., t)\right\|_{2}^{4}\left[\nu^{2} \delta-4 t c_{4} c_{3}\left\|A \boldsymbol{v}^{n}(., 0)\right\|_{2}^{4}\right] \leq \nu^{2} \delta\left\|A \boldsymbol{v}^{n}(., 0)\right\|_{2}^{4}
$$

for $t \in\left(0, T^{*}\right)$ where $T^{*}$ satisfies

$$
0<T^{*}<\frac{\nu^{2} \delta}{4 c_{4} c_{3}\left\|A \boldsymbol{v}^{n}(., 0)\right\|_{2}^{4}} .
$$

Suppose further that $T_{1}^{*}$ is a fixed number which is chosen so that

$$
0<T_{1}^{*}<\frac{\nu^{2} \delta}{4 c_{4} c_{3}\left\|A \boldsymbol{v}_{0}\right\|_{2}^{4}} .
$$

Then $T_{1}^{*}$ also satisfies (25) because

$$
\left\|A \boldsymbol{v}^{n}(., 0)\right\|_{2}^{2}=\sum_{i \in Z_{n}^{*}} \lambda_{i}^{2} a_{i}^{2}(0)=\sum_{i \in Z_{n}^{*}} \lambda_{i}^{2}\left(\boldsymbol{v}_{0}, \boldsymbol{e}^{i}\right)_{2}^{2} \leq \sum_{i \in Z^{*}} \lambda_{i}^{2}\left(\boldsymbol{v}_{0}, \boldsymbol{e}^{i}\right)_{2}^{2}=\left\|A \boldsymbol{v}_{0}\right\|_{2}^{2} .
$$

Thus, the integration of inequality (23) provides the estimates

$$
\begin{aligned}
& \left\|A \boldsymbol{v}^{n}(., t)\right\|_{2} \leq c_{5} \quad \text { for } t \in\left(0, T_{1}^{*}\right), \\
& \int_{0}^{T_{1}^{*}}\left\|A^{2} \boldsymbol{v}^{n}(., t)\right\|_{2}^{2} \mathrm{~d} t \leq c_{6}
\end{aligned}
$$

where $c_{5}$ and $c_{6}$ are independent of $n$. The proof of the part of the lemma corresponding to $j=1$ can now be completed by a standard limit procedure.

Let us further suppose that $\boldsymbol{v}_{0} \in D^{2}$. Multiplying the $i$-th equation in (19) by $\lambda_{i}^{4} a_{i}$ and summing over $i \in \mathbf{Z}_{n}^{*}$, we obtain the equation whose equivalent form is

$$
\frac{\mathrm{d}}{\mathrm{d} t} \frac{1}{2} \int_{\Omega}\left|A^{2} \boldsymbol{v}^{n}\right|^{2} \mathrm{~d} \boldsymbol{x}+\int_{\Omega}\left(A \boldsymbol{v}^{n} \times \boldsymbol{v}^{n}\right) \cdot A^{4} \boldsymbol{v}^{n} \mathrm{~d} \boldsymbol{x}+\nu \int_{\Omega}\left|A^{3} \boldsymbol{v}^{n}\right|^{2} \mathrm{~d} \boldsymbol{x}=0 .
$$

The second integral can be treated as follows:

$$
\int_{\Omega}\left(A \boldsymbol{v}^{n} \times \boldsymbol{v}^{n}\right) \cdot A^{4} \boldsymbol{v}^{n} \mathrm{~d} \boldsymbol{x}=\int_{\partial \Omega}\left(A \boldsymbol{v}^{n} \times \boldsymbol{v}^{n}\right) \cdot\left(\boldsymbol{n} \times A^{3} \boldsymbol{v}^{n}\right) \mathrm{d} S+\int_{\Omega} A\left(A \boldsymbol{v}^{n} \times \boldsymbol{v}^{n}\right) \cdot A^{3} \boldsymbol{v}^{n} \mathrm{~d} \boldsymbol{x}
$$
$A \boldsymbol{v}^{n}$ and $\boldsymbol{v}^{n}$ are tangential a.e. on $\partial \Omega$, so $A \boldsymbol{v}^{n} \times \boldsymbol{v}^{n}$ is normal. On the other hand, $\boldsymbol{n} \times A^{3} \boldsymbol{v}^{n}$ is tangential. This means that $\left(A \boldsymbol{v}^{n} \times \boldsymbol{v}^{n}\right) \cdot\left(\boldsymbol{n} \times A^{3} \boldsymbol{v}^{n}\right)=0$ a.e. on $\partial \Omega$ and the surface integral equals zero. Hence

$$
\begin{aligned}
& \int_{\Omega}\left(A \boldsymbol{v}^{n} \times \boldsymbol{v}^{n}\right) \cdot A^{4} \boldsymbol{v}^{n} \mathrm{~d} \boldsymbol{x}=\int_{\Omega} A\left(A \boldsymbol{v}^{n} \times \boldsymbol{v}^{n}\right) \cdot A^{3} \boldsymbol{v}^{n} \mathrm{~d} \boldsymbol{x} \\
& =\int_{\Omega}\left[\left(\boldsymbol{v}^{n} \cdot \nabla\right) A \boldsymbol{v}^{n}-\left(A \boldsymbol{v}^{n} \cdot \nabla\right) \boldsymbol{v}^{n}\right] \cdot A^{3} \boldsymbol{v}^{n} \mathrm{~d} \boldsymbol{x} \\
& \leq \frac{\nu}{4} \int_{\Omega}\left|A^{3} \boldsymbol{v}^{n}\right|^{2} \mathrm{~d} \boldsymbol{x}+\frac{c_{7}}{\nu} \int_{\Omega}\left(\left|\boldsymbol{v}^{n}\right|^{2}\left|\nabla A \boldsymbol{v}^{n}\right|^{2}+\left|A \boldsymbol{v}^{n}\right|^{2}\left|\nabla \boldsymbol{v}^{n}\right|^{2}\right) \mathrm{d} \boldsymbol{x} \\
& \leq \frac{\nu}{4}\left\|A^{3} \boldsymbol{v}^{n}\right\|_{2}^{2}+\frac{c_{8}}{\nu}\left(\int_{\Omega}\left|\boldsymbol{v}^{n}\right| \mathrm{d} \boldsymbol{x}\right)^{1 / 3}\left(\int_{\Omega}\left|\nabla A \boldsymbol{v}^{n}\right|^{6} \mathrm{~d} \boldsymbol{x}\right)^{1 / 6}\left(\int_{\Omega}\left|\nabla A \boldsymbol{v}^{n}\right|^{2} \mathrm{~d} \boldsymbol{x}\right)^{1 / 2}
\end{aligned}
$$




$$
\begin{aligned}
& +\frac{c_{8}}{\nu}\left(\int_{\Omega}\left|A \boldsymbol{v}^{n}\right|^{6} \mathrm{~d} \boldsymbol{x}\right)^{1 / 3}\left(\int_{\Omega}\left|\nabla \boldsymbol{v}^{n}\right|^{6} \mathrm{~d} \boldsymbol{x}\right)^{1 / 6}\left(\int_{\Omega}\left|\nabla \boldsymbol{v}^{n}\right|^{2} \mathrm{~d} \boldsymbol{x}\right)^{1 / 2} \\
\leq & \frac{\nu}{4}\left\|A^{3} \boldsymbol{v}^{n}\right\|_{2}^{2}+\frac{c_{9}}{\nu}\left\|A \boldsymbol{v}^{n}\right\|_{2}^{2}\left\|A^{3} \boldsymbol{v}^{n}\right\|_{2}\left\|A^{2} \boldsymbol{v}^{n}\right\|_{2}+\frac{c_{9}}{\nu}\left\|A^{2} \boldsymbol{v}^{n}\right\|_{2}^{3}\left\|A \boldsymbol{v}^{n}\right\|_{2} \\
\leq & \frac{\nu}{2}\left\|A^{3} \boldsymbol{v}^{n}\right\|_{2}^{2}+\frac{c_{10} c_{5}^{4}}{\nu^{2}}\left\|A^{2} \boldsymbol{v}^{n}\right\|_{2}^{2}+\frac{c_{11} c_{5}}{\nu}\left\|A^{2} \boldsymbol{v}^{n}\right\|_{2}^{3} .
\end{aligned}
$$

(We have estimated $\left\|A \boldsymbol{v}^{n}\right\|_{2}$ by means of (27).) Substituting these estimates into (29), we obtain:

$$
\frac{\mathrm{d}}{\mathrm{d} t} \frac{1}{2}\left\|A^{2} \boldsymbol{v}^{n}\right\|_{2}^{2}+\frac{\nu}{2}\left\|A^{3} \boldsymbol{v}^{n}\right\|_{2}^{2} \leq \frac{c_{10} c_{5}^{4}}{\nu^{2}}\left\|A^{2} \boldsymbol{v}^{n}\right\|_{2}^{2}+\frac{c_{11} c_{5}}{\nu}\left\|A^{2} \boldsymbol{v}^{n}\right\|_{2}^{3} .
$$

Integrating this inequality, we can show that if $T_{2}^{*}$ is chosen so that $0<T_{2}^{*} \leq T_{1}^{*}$ and

$$
T_{2}^{*}<c_{12}\left[\frac{\pi}{2}-\arctan \left(\frac{1}{2}\left\|A^{2} \boldsymbol{v}_{0}\right\|_{2}^{2}\right)\right]
$$

then

$$
\begin{aligned}
& \left\|A^{2} \boldsymbol{v}^{n}(., t)\right\|_{2} \leq c_{13} \quad \text { for } t \in\left(0, T_{2}^{*}\right), \\
& \int_{0}^{T_{1}^{*}}\left\|A^{3} \boldsymbol{v}^{n}(., t)\right\|_{2}^{2} \mathrm{~d} t \leq c_{14}
\end{aligned}
$$

where $c_{12}, c_{13}$ and $c_{14}$ are appropriate constants independent of $n$. A standard limit procedure now again enables to complete the proof.

REMARK 1. The inclusion $A^{j+1} \boldsymbol{v} \in L^{2}\left(0, T_{j}^{*} ; L_{\sigma}^{2}(\Omega)^{3}\right)$ in Theorem 5 , in the case of $j=2$, says that $\boldsymbol{v}(., t) \in D\left(A^{3}\right) \equiv D^{3}$ for a.a. $t \in\left(0, T_{2}^{*}\right)$. This means that $\boldsymbol{v}(., t)$ satisfies not only the three boundary conditions in (14), but also

$$
A^{3} \boldsymbol{v}(., t) \cdot \boldsymbol{n}=0 \quad \text { on } \partial \Omega .
$$

This is in agreement with our result from [1] where we have shown that if $\boldsymbol{v}$ satisfies (14) then $\boldsymbol{\omega}=\operatorname{curl} \boldsymbol{v}$ also satisfies (14). Thus, $A^{2} \boldsymbol{\omega}(., t) \cdot \boldsymbol{n}=-\Delta \boldsymbol{\omega}(., t) \cdot \boldsymbol{n}=0$ on $\partial \Omega$.

By analogy with J. Leray [7] and with G. P. Galdi [4], let us call an epoch of irregularity of weak solution $\boldsymbol{v}$ to the problem (1)-(4) an instant of time $\vartheta$ such that $\boldsymbol{v}(., t)$ is (after a possible re-definition on a set of measure zero in $Q_{T}$ ) an element of $D^{1}$ continuously depending on $t$ on the interval $(\vartheta-\epsilon, \vartheta)$ for some $\epsilon>0$ and $\|A \boldsymbol{v}(., t)\|_{2} \rightarrow+\infty$ as $t \rightarrow \vartheta_{-}$. Obviously, the set of all epochs of irregularity of $\boldsymbol{v}$ is at most countable. If $\boldsymbol{v}(., t) \in D^{1}$, denote by $\vartheta(t)$ the least epoch of irregularity of $\boldsymbol{v}$, greater than $t$. Theorem 5 guarantees that either such an epoch of irregularity exists in $(t, T)$ or $\|A \boldsymbol{v}\|_{2}$ is bounded (as a function of time) on $(t, T)$. In the latter case, we put $\vartheta(t)=T$.

TheOREM 6. Suppose that $\boldsymbol{v}$ is a weak solution to the problem (1)-(4) that satisfies the strong energy inequality, i.e.

$$
\|\boldsymbol{v}(., t)\|_{2}^{2}+2 \nu \int_{\xi}^{t}\|\nabla \boldsymbol{v}(., \xi)\|_{2}^{2} \mathrm{~d} \xi \leq\|\boldsymbol{v}(., \xi)\|_{2}^{2}
$$

for a.a. $\xi \in(0, T)$ and all $t \in[\xi, T)$. Then,

$$
(0, T)=\bigcup_{\gamma \in \Gamma}\left(a_{\gamma}, b_{\gamma}\right) \cup G
$$


where set $\Gamma$ is countable, $\left(a_{\gamma}, b_{\gamma}\right)$ are non-overlapping intervals and $\boldsymbol{v}$, after a possible re-definition on a set of measure zero, has the following properties:

1. $\boldsymbol{v}(., t)$ is an element of $D^{2}$, continuously depending on $t$ on each of the intervals $\left(a_{\gamma}, b_{\gamma}\right)$,

2. $A^{3} \boldsymbol{v} \in L_{l o c}^{2}\left(a_{\gamma}, b_{\gamma} ; L_{\sigma}^{2}(\Omega)^{3}\right)$ for all $\gamma \in \Gamma$,

3. $\partial A \boldsymbol{v} / \partial t \in L_{l o c}^{2}\left(a_{\gamma}, b_{\gamma} ; L_{\sigma}^{2}(\Omega)^{3}\right)$ for all $\gamma \in \Gamma$,

4. set $G$ has the $\frac{1}{2}$-dimensional Hausdorff measure equal to zero,

5. the strong energy inequality (35) is satisfied for all $\xi \in\left(a_{\gamma}, b_{\gamma}\right)(\gamma \in \Gamma)$ and all $t \in[\xi, T)$,

6. each of the points $b_{\gamma}($ for $\gamma \in \Gamma)$ either coincides with $T$ or is an epoch of irregularity of $\boldsymbol{v}$.

Proof. Since $\boldsymbol{v} \in L^{2}\left(0, T ; D^{1}\right)$, there exists $M_{1} \subset(0, T)$ such that $M_{1}$ is of measure zero and $\boldsymbol{v}(., t) \in D^{1}$ for all $t \in(0, T)-M_{1}$. Denote by $M_{2}$ the set of such time instants from $(0, T)$ that the inequality (35) is satisfied for all $\xi \in(0, T)-M_{2}$ and all $\left.\left.t \in\right] \xi, T\right) . M_{2}$ is of measure zero, too. Suppose that $\xi \in(0, T)-\left(M_{1} \cup M_{2}\right)$. Due to Theorem 5 , the problem (1)-(4) has a strong solution on each interval $\left(\xi, t^{*}\right)$ where $\xi<t^{*}<\vartheta(\xi)$. Since $\boldsymbol{v}$ is assumed to satisfy the energy inequality (35), we can identify it with the strong solution on the interval $\left(\xi, t^{*}\right)$. (See the theorem on uniqueness in [1], Theorem 5.2.) The union

$$
J=\bigcup_{t \in(0, T)-\left(M_{1} \cup M_{2}\right)}(t, \vartheta(t))
$$

consists of a countable number of disjoint open intervals $\left(a_{\gamma}, b_{\gamma}\right) ; \gamma \in \Gamma$. Denote by $M_{3}$ the set of epochs of irregularity of solution $\boldsymbol{v}$ in $(0, T)$ and by $M_{4}$ the set of time instants $a_{\gamma}$ for $\gamma \in \Gamma$. Obviously, the measure of $M_{3}$ and $M_{4}$ is zero. Put $G=(0, T)-J$. $G$ can contain only points from $M_{1} \cup M_{2} \cup M_{3} \cup M_{4}$. This implies that $G$ is of measure zero.

The properties $1-3$ of solution $\boldsymbol{v}$ on each of the intervals $\left(a_{\gamma}, b_{\gamma}\right)$ easily follow from Theorem 5 and from the steps of its proof, especially from the way how numbers $T_{1}^{*}$ and $T_{2}^{*}$ could be chosen. (See (26) and (31).)

A similar statement to our statement 4 is well known from the case of the Dirichlet boundary condition. Thus, we do not show the proof here. We refer e.g. to G. P. Galdi [4], proof of Theorem 6.4, for the basic ideas and steps.

The statement about the strong energy inequality in item 5 can be proved in this way: We suppose that $\xi \in\left(a_{\gamma}, b_{\gamma}\right)$. We can construct a weak solution $\boldsymbol{w}$ on the interval $(\xi, T)$ which satisfies the energy inequality (35) for all $t \in[\xi, T)$ and which coincides with $\boldsymbol{v}$ at time $\xi$. (See the remark below.) Due to the theorem on uniqueness, $\boldsymbol{w}(., t)=\boldsymbol{v}(., t)$ for $t \in\left(\xi, b_{\gamma}\right)$. Hence we can deduce that $\boldsymbol{v}$ also satisfies (35) for all $t \in[\xi, T)$.

Item 6 can easily be proved by contradiction.

REMARK 2. It is known from the theory of the Navier-Stokes equation with the Dirichlet boundary condition (5) that a weak solution can be constructed so that it satisfies the strong energy inequality (35). It is not known for an arbitrary domain $\Omega$, however the result is true in the special case when $\Omega$ is a cube. Considering now the boundary conditions (4) instead of (5), we can repeat all important steps of the proof (see e.g. [4]) and we can find out that the weak solution $\boldsymbol{v}$ can also be constructed so that it satisfies 
(35). Thus, the assumption about the strong energy inequality in Theorem 6 does not represent a serious restriction.

3. Estimates up to the boundary. The initial-boundary value problem (1)-(3), (5), localized to the interior of $\Omega$, which was studied in our papers [9] and [10], had the advantage that a localized velocity and all its derivatives differed from zero only in a small cylinder $C$ around singular point $\left(\boldsymbol{x}_{0}, \vartheta\right)$. It enabled one to perform arbitrarily the integration by parts and not to care about the integrals on the boundary. Theorem 1 only provides the information on local regularity of solution $\boldsymbol{v}$ in $C$ and consequently in any subdomain $D$ of $Q_{T}$, but it does not say anything about the regularity of $\boldsymbol{v}$ up to the boundary of $\Omega$.

In this section, we assume that $\boldsymbol{v}$ is a weak solution of the problem (13), (2), (3) with the boundary conditions (14). We recall that $\Omega$ is the cube $(-\pi, \pi)^{3}$. The following theorem provides a similar information as Theorem 1 , however on the whole domain $\Omega . \sigma$ again denotes the rate of deformation tensor (i.e. $\sigma=\frac{1}{2}\left[\nabla \boldsymbol{v}+(\nabla \boldsymbol{v})^{T}\right]$ ) and $\left(\zeta_{2}\right)_{+}$means the positive part of $\zeta_{2}$.

THEOREM 7. Let $\boldsymbol{v}$ be a weak solution to the problem (1)-(4) that satisfies the strong energy inequality (35) for a.a. $\xi \in(0, T)$ and for all $t \in[\xi, T)$. Let $\zeta_{1} \leq \zeta_{2} \leq \zeta_{3}$ be the eigenvalues of tensor $\sigma$. Suppose that $0 \leq t_{1}<t_{2} \leq T$ and

(ii) one of the functions $\zeta_{1},\left(\zeta_{2}\right)_{+}, \zeta_{3}$ belongs to $L^{r}\left(t_{1}, t_{2} ; L^{s}(\Omega)\right)$ for some real numbers $r, s$ such that $1 \leq r \leq+\infty, 3 / 2<s \leq+\infty$ and $2 / r+3 / s \leq 2$.

Then $\boldsymbol{v} \in C\left(t_{1}+\delta, t_{2}-\delta ; D^{2}\right), A^{3} \boldsymbol{v} \in L^{2}\left(t_{1}+\delta, t_{2}-\delta ; L_{\sigma}^{2}(\Omega)^{3}\right)$ and $\partial A \boldsymbol{v} / \partial t \in L^{2}\left(t_{1}+\right.$ $\left.\delta, t_{2}-\delta ; L_{\sigma}^{2}(\Omega)^{3}\right)$ for each $\delta>0$ such that $t_{1}+\delta<t_{2}-\delta$.

Proof. Suppose that an epoch of irregularity $b_{\gamma}$ (for some $\gamma \in \Gamma$ ) belongs to the interval $\left(t_{1}, t_{2}\right)$.

Item 4 of Theorem 6 and Lemma 4 imply that $\boldsymbol{v}(., t) \in W^{3,2}(\Omega)^{3}$ for $t \in\left(a_{\gamma}, b_{\gamma}\right)-M_{5}$ where $M_{5}$ is a set of measure zero. Suppose that $t \in\left(t_{1}, t_{2}\right) \cap\left(a_{\gamma}, b_{\gamma}\right)-M_{5}$. Then the first order space partial derivatives of $\boldsymbol{v}(., t)$ can be extended from $\Omega$ to $\partial \Omega$ as continuous functions on $\bar{\Omega}$. (See e.g. J. Nečas [8], p. 72.) The condition $A^{2} \boldsymbol{v} \cdot \boldsymbol{n}=0$ a.e. on $\partial \Omega$ means that $\Delta \boldsymbol{v} \cdot \boldsymbol{n}=0$ on $\partial \Omega$ and so $\Delta \boldsymbol{v}=\Delta P_{\sigma} \boldsymbol{v}=P_{\sigma} \Delta \boldsymbol{v}$. Thus, multiplying the Navier-Stokes equation by $\Delta \boldsymbol{v}$, integrating on $\Omega$ and using the selfadjointness of the operator $A$, we obtain:

$$
\frac{\mathrm{d}}{\mathrm{d} t} \frac{1}{2} \int_{\Omega}|A \boldsymbol{v}|^{2} \mathrm{~d} \boldsymbol{x}+\nu \int_{\Omega}|\Delta \boldsymbol{v}|^{2} \mathrm{~d} \boldsymbol{x}=\int_{\Omega}(\boldsymbol{v} \cdot \nabla) \boldsymbol{v} \cdot \Delta \boldsymbol{v} \mathrm{d} \boldsymbol{x} .
$$

The integral on the right-hand side can be treated in this way:

$$
\begin{aligned}
& \int_{\Omega}(\boldsymbol{v} \cdot \nabla) \boldsymbol{v} \cdot \Delta \boldsymbol{v} \mathrm{d} \boldsymbol{x}=\int_{\Omega} v_{j}\left(\partial_{j} v_{i}\right)\left(\partial_{k}^{2} v_{i}\right) \mathrm{d} \boldsymbol{x}=\int_{\partial \Omega} v_{j}\left(\partial_{j} v_{i}\right)\left(\partial_{k} v_{i}\right) n_{k} \mathrm{~d} S \\
& -\int_{\Omega}\left(\partial_{j} v_{i}\right)\left(\partial_{k} v_{i}\right)\left(\partial_{k} v_{j}\right) \mathrm{d} \boldsymbol{x} .
\end{aligned}
$$

$\left(\partial_{i}\right.$ denotes the partial derivative with respect to $x_{i}$.) We are further going to show that the integral on $\partial \Omega$ equals zero. 
$\partial \Omega$ consists of 6 squares, let us denote them by $S_{1}, \ldots, S_{6}$. We can assume that the squares are numbered so that $S_{1}=\left\{\boldsymbol{x} \equiv\left(x_{1}, x_{2}, x_{3}\right) ;-\pi \leq x_{1} \leq \pi,-\pi \leq x_{2} \leq \pi, x_{3}=\right.$ $-\pi\}$ and $S_{2}=\left\{\boldsymbol{x} \equiv\left(x_{1}, x_{2}, x_{3}\right) ;-\pi \leq x_{1} \leq \pi, x_{2}=-\pi,-\pi \leq x_{3} \leq \pi\right\}$. The sides of square $S_{1}$ are four line segments - let us denote them by $l_{1}^{1}, \ldots, l_{1}^{4}$ where for example $l_{1}^{1}$ connects the points $(-\pi,-\pi,-\pi)$ and $(\pi,-\pi,-\pi)$. Then for $\boldsymbol{x}=\left(x_{1}, x_{2},-\pi\right) \in S_{1}$, we have: $\boldsymbol{n}=(0,0,-1), v_{3}=0$ and $\partial_{1} v_{3}=\partial_{2} v_{3}=0$. Moreover, if we use the fact that $\boldsymbol{v}$ coincides with a potential vector field $\nabla \varphi$ on $\partial \Omega$ (following from (8)) then we also have: $v_{j}=\partial_{j} \varphi$ and $\partial_{j} v_{i}=\partial_{j} \partial_{i} \varphi$ for $i, j=1,2$. Thus,

$\int_{S_{1}} v_{j}\left(\partial_{j} v_{i}\right)\left(\partial_{k} v_{i}\right) n_{k} \mathrm{~d} S=-\int_{S_{1}} \sum_{i, j=1}^{2}\left(\partial_{j} \varphi\right)\left(\partial_{i} \partial_{j} \varphi\right)\left(\partial_{3} v_{i}\right) \mathrm{d} S=-\int_{S_{1}} \sum_{i=1}^{2}\left(\partial_{i} \Phi\right)\left(\partial_{3} v_{i}\right) \mathrm{d} S$

where $\Phi=\frac{1}{2}\left[\left(\partial_{1} \varphi\right)^{2}+\left(\partial_{2} \varphi\right)^{2}\right]=\frac{1}{2}|\nabla \varphi|^{2}$ because $\partial_{3} \varphi=v_{3}=0$ on $S_{1}$. Denote by $\boldsymbol{\nu}=\left(\nu_{1}, \nu_{2}\right)$ the outer normal vector on the boundary of $S_{1}$. We have:

$$
\begin{aligned}
& \int_{S_{1}} v_{j}\left(\partial_{j} v_{i}\right)\left(\partial_{k} v_{i}\right) n_{k} \mathrm{~d} S \\
& =-\int_{\partial S_{1}} \Phi\left[\left(\partial_{3} v_{1}\right) \nu_{1}+\left(\partial_{3} v_{2}\right) \nu_{2}\right] \mathrm{d} l+\int_{S_{1}} \Phi\left[\left(\partial_{1} \partial_{3} v_{1}\right)+\left(\partial_{2} \partial_{3} v_{2}\right)\right] \mathrm{d} x_{1} \mathrm{~d} x_{2} \\
& =-\int_{\partial S_{1}} \Phi\left[\left(\partial_{3} v_{1}\right) \nu_{1}+\left(\partial_{3} v_{2}\right) \nu_{2}\right] \mathrm{d} l=-\left(\int_{l_{1}^{1}}+\ldots+\int_{l_{1}^{4}}\right) \ldots
\end{aligned}
$$

because $\left(\partial_{1} \partial_{3} v_{1}\right)+\left(\partial_{2} \partial_{3} v_{2}\right)=-\partial_{3}^{2} v_{3}$ and it is equal to zero on $S_{1}$ (due to the third boundary condition in (4)). The integral on $l_{1}^{1}$ equals

$$
\int_{-\pi}^{\pi}\left[-\Phi\left(\partial_{3} v_{2}\right)\right] \mathrm{d} x_{1}
$$

because $\nu_{1}=0, \nu_{2}=-1$ and $\mathrm{d} l=\mathrm{d} x_{1}$ on $l_{1}^{1}$. However, the edge $l_{1}^{1}$ is also one of the sides of the square $S_{2}$ where $v_{2}$ and $\partial_{3} v_{2}$ are equal to zero. Hence, by continuity, $\partial_{3} v_{2}=0$ on $l_{1}^{1}$ and the integral in (41) equals zero. By analogy, the line integrals on $l_{1}^{2}, l_{1}^{3}$ and $l_{1}^{4}$ are also equal to zero. Hence the surface integral on the left-hand side of (40) is also zero and again by analogy, the same surface integrals on squares $S_{2}, \ldots, S_{6}$ are also equal to zero. Thus, we have shown that the surface integral in (39) equals zero.

Let us denote by $\tau$ the skew-symmetric part of $\nabla \boldsymbol{v}$. (Recall that $\sigma$ is the symmetric part.) $\boldsymbol{\omega} \equiv\left(\omega_{1}, \omega_{2}, \omega_{3}\right)$ will further denote the vorticity, i.e. $\boldsymbol{\omega}=A \boldsymbol{v}$. The last integral on the right-hand side of (39) can be written in this way:

$$
\begin{aligned}
& \int_{\Omega}\left(\partial_{j} v_{i}\right)\left(\partial_{k} v_{i}\right)\left(\partial_{k} v_{j}\right) \mathrm{d} \boldsymbol{x}=\int_{\Omega} \sigma_{k j}\left(\partial_{j} v_{i}\right)\left(\partial_{k} v_{i}\right) \mathrm{d} \boldsymbol{x}=\int_{\Omega} \sigma_{k j}\left(\sigma_{i j}+\tau_{i j}\right)\left(\sigma_{i k}+\tau_{i k}\right) \mathrm{d} \boldsymbol{x} \\
& =\int_{\Omega} \sigma_{k j} \sigma_{i j} \sigma_{i k} \mathrm{~d} \boldsymbol{x}+\int_{\Omega} \sigma_{k j} \tau_{i j} \tau_{i k} \mathrm{~d} \boldsymbol{x}=\int_{\Omega} \sigma_{j k} \sigma_{k i} \sigma_{i j} \mathrm{~d} \boldsymbol{x}-\frac{1}{4} \int_{\Omega} \sigma_{k j} \omega_{k} \omega_{j} \mathrm{~d} \boldsymbol{x} .
\end{aligned}
$$

Substituting this to (39) and (38), we get:

$$
\frac{\mathrm{d}}{\mathrm{d} t} \frac{1}{2} \int_{\Omega}|A \boldsymbol{v}|^{2} \mathrm{~d} \boldsymbol{x}+\nu \int_{\Omega}\left|A^{2} \boldsymbol{v}\right|^{2} \mathrm{~d} \boldsymbol{x}=-\int_{\Omega} \sigma_{j k} \sigma_{k i} \sigma_{i j} \mathrm{~d} \boldsymbol{x}+\frac{1}{4} \int_{\Omega} \sigma_{k j} \omega_{k} \omega_{j} \mathrm{~d} \boldsymbol{x} .
$$


Applying operator curl to equation (1) and multiplying the equation by $\boldsymbol{\omega}$, we obtain:

$$
\frac{\mathrm{d}}{\mathrm{d} t} \frac{1}{2} \int_{\Omega}|\boldsymbol{\omega}|^{2} \mathrm{~d} \boldsymbol{x}-\nu \int_{\Omega} \Delta \boldsymbol{\omega} \cdot \boldsymbol{\omega} \mathrm{d} \boldsymbol{x}=\int_{\Omega}(\boldsymbol{\omega} \cdot \nabla) \boldsymbol{v} \cdot \boldsymbol{\omega} \mathrm{d} \boldsymbol{x}=\int_{\Omega} \sigma_{k j} \omega_{k} \omega_{j} \mathrm{~d} \boldsymbol{x} .
$$

Using the identities

$$
-\nu \int_{\Omega} \Delta \boldsymbol{\omega} \cdot \boldsymbol{\omega} \mathrm{d} \boldsymbol{x}=\nu \int_{\Omega} A^{3} \boldsymbol{v} \cdot A \boldsymbol{v} \mathrm{d} \boldsymbol{x}=\nu \int_{\Omega} A^{2} \boldsymbol{v} \cdot A^{2} \boldsymbol{v} \mathrm{d} \boldsymbol{x},
$$

we can rewrite (44) in the form

$$
\frac{\mathrm{d}}{\mathrm{d} t} \frac{1}{2} \int_{\Omega}|A \boldsymbol{v}|^{2} \mathrm{~d} \boldsymbol{x}+\nu \int_{\Omega}|\Delta \boldsymbol{v}|^{2} \mathrm{~d} \boldsymbol{x}=\int_{\Omega} \sigma_{k j} \omega_{k} \omega_{j} \mathrm{~d} \boldsymbol{x} .
$$

Multiplying (45) by $\frac{1}{4}$ and subtracting it from (43), we obtain:

$$
\frac{\mathrm{d}}{\mathrm{d} t} \frac{3}{8} \int_{\Omega}|A \boldsymbol{v}|^{2} \mathrm{~d} \boldsymbol{x}+\frac{3 \nu}{4} \int_{\Omega}\left|A^{2} \boldsymbol{v}\right|^{2} \mathrm{~d} \boldsymbol{x}=-\int_{\Omega} \sigma_{j k} \sigma_{k i} \sigma_{i j} \mathrm{~d} \boldsymbol{x} .
$$

$\sigma_{j k} \sigma_{k i} \sigma_{i j}$ equals $\operatorname{tr}\left[\sigma^{3}\right]$ (the trace of $\sigma^{3}$ ). The system of coordinates can be, at each point $(\boldsymbol{x}, t)$, chosen so that $\sigma^{3}$ has a diagonal representation with $\zeta_{1}^{3}, \zeta_{2}^{3}$ and $\zeta_{3}^{3}$ on the diagonal. Hence $\operatorname{tr}\left[\sigma^{3}\right]=\zeta_{1}^{3}+\zeta_{2}^{3}+\zeta_{3}^{3}$ and this equality holds independently of the choice of the coordinate system because $\operatorname{tr}\left[\sigma^{3}\right]$ and $\zeta_{1}^{3}, \zeta_{2}^{3}$ and $\zeta_{3}^{3}$ are invariants of $\sigma^{3}$. Moreover, using the identity $\operatorname{tr}[\sigma]=\zeta_{1}+\zeta_{2}+\zeta_{3}=0$ (which is nothing else than the equation of continuity (2)), we get: $\operatorname{tr}\left[\sigma^{3}\right]=3 \zeta_{1} \zeta_{2} \zeta_{3}$. Thus,

$$
\frac{\mathrm{d}}{\mathrm{d} t} \frac{3}{8} \int_{\Omega}|A \boldsymbol{v}|^{2} \mathrm{~d} \boldsymbol{x}+\frac{3 \nu}{4} \int_{\Omega}\left|A^{2} \boldsymbol{v}\right|^{2} \mathrm{~d} \boldsymbol{x}=-3 \int_{\Omega} \zeta_{1} \zeta_{2} \zeta_{3} \mathrm{~d} \boldsymbol{x} \leq 3 \int_{\Omega}\left(-\zeta_{1}\right)\left(\zeta_{2}\right)_{+} \zeta_{3} \mathrm{~d} \boldsymbol{x}
$$

Suppose that $\left(\zeta_{2}\right)_{+}$satisfies the assumptions of condition (ii). (The other cases mentioned in (ii) could be treated similarly.) Suppose further that $t_{0}, t_{0}+\epsilon \in\left(a_{\gamma}, b_{\gamma}\right) \cap\left(t_{1}, t_{2}\right)-$ $M_{5}$. We shall further integrate (47) from $t_{0}$ to $t_{0}+\epsilon$. We shall apply the inequality

$$
\|g\|_{L^{a}\left(\alpha, \beta ; L^{b}(\Omega)\right)} \leq\|g\|_{L^{\infty}\left(\alpha, \beta ; L^{2}(\Omega)\right)}^{2 / a+3 / b-3 / 2}\|g\|_{L^{2}\left(\alpha, \beta ; L^{6}(\Omega)\right)}^{5 / 2-(2 / a+3 / b)}
$$

which is valid for $g \in L^{\infty}\left(\alpha, \beta ; L^{2}(\Omega)\right) \cap L^{2}\left(\alpha, \beta ; L^{6}(\Omega)\right), 2 \leq a \leq+\infty, 2 \leq b \leq 6$ and $\frac{3}{2} \leq 2 / a+3 / b \leq \frac{5}{2}$ and which can be derived by means of the Hölder inequality. We obtain

$$
\begin{aligned}
& \|A \boldsymbol{v}\|_{L^{\infty}\left(t_{0}, t_{0}+\epsilon ; L^{2}(\Omega)^{3}\right)}^{2}+2 \nu\left\|A^{2} \boldsymbol{v}\right\|_{L^{2}\left(t_{0}, t_{0}+\epsilon ; L^{2}(\Omega)^{3}\right)}^{2} \leq 8 \int_{t_{0}}^{t_{0}+\epsilon} \int_{\Omega}\left(-\zeta_{1}\right)\left(\zeta_{2}\right)_{+} \zeta_{3} \mathrm{~d} \boldsymbol{x} \mathrm{d} \xi \\
& \leq c_{15}\left\|\left(\zeta_{2}\right)_{+}\right\|_{L^{r}\left(t_{0}, t_{0}+\epsilon ; L^{s}(\Omega)\right)}\left[\int_{t_{0}}^{t_{0}+\epsilon}\left(\int_{\Omega}\left|\zeta_{1} \zeta_{3}\right|^{\frac{2 s}{s-1}} \mathrm{~d} \boldsymbol{x}\right)^{\frac{s-1}{2 s} \frac{2 r}{r-1}} \mathrm{~d} \xi\right]^{\frac{r-1}{2 r}} \\
& \leq c_{16}\left\|\left(\zeta_{2}\right)_{+}\right\|_{L^{r}\left(t_{0}, t_{0}+\epsilon ; L^{s}(\Omega)\right)} \\
& \quad \cdot\left(\|A \boldsymbol{v}\|_{L^{\infty}\left(t_{0}, t_{0}+\epsilon ; L^{2}(\Omega)^{3}\right)}^{2}+2 \nu\left\|A^{2} \boldsymbol{v}\right\|_{L^{2}\left(t_{0}, t_{0}+\epsilon ; L^{2}(\Omega)^{3}\right)}^{2}\right)^{\frac{2 / r+3 / s}{2}} .
\end{aligned}
$$

We have used the inequality $-\zeta_{1} \zeta_{3} \leq c_{17}|\nabla \boldsymbol{v}|^{2}$. If we choose $t_{0}$ so close to $b_{\gamma}$ that

$$
c_{16}\left\|\left(\zeta_{2}\right)_{+}\right\|_{L^{r}\left(t_{0}, b_{\gamma} ; L^{s}(\Omega)\right)}<1
$$

we obtain

$$
\|A \boldsymbol{v}\|_{L^{\infty}\left(t_{0}, t_{0}+\epsilon ; L^{2}(\Omega)^{3}\right)}^{2}+2 \nu\left\|A^{2} \boldsymbol{v}\right\|_{L^{2}\left(t_{0}, t_{0}+\epsilon ; L^{2}(\Omega)^{3}\right)}^{2} \leq c_{18}
$$


where constant $c_{18}$ is independent of $\epsilon$. The estimate holds for $t_{0}+\epsilon$ being arbitrarily close to $b_{\gamma}$. Using Theorem 5 (on the local in time existence of a strong solution) and the theorem on uniqueness (see [1], Theorem 5.2), we can show that solution $\boldsymbol{v}$ can be prolonged, as a strong solution, from the interval $\left(a_{\gamma}, b_{\gamma}\right)$ over $b_{\gamma}$ further to the right. This is the contradiction with the assumption that $b_{\gamma}$ is the epoch of irregularity. The desired regularity of $\boldsymbol{v}$ on the time interval $\left(t_{1}, t_{2}\right)$ now follows from Theorem 6 .

Acknowledgements. The research was supported by the University of Sud-Toulon-Var and in the case of the first author, it was also supported by the Grant Agency of the Czech Republic (grant No. 201/05/0005) and by the research plan of the Ministry of Education of the Czech Republic No. MSM 6840770010. The second author wishes to express his gratitude to the Banach Center for the hospitality and good working conditions during his stay there.

\section{References}

[1] H. Bellout, J. Neustupa and P. Penel, On the Navier-Stokes equation with boundary conditions based on vorticity, Math. Nachr. 269-270 (2004), 59-72.

[2] L. Caffarelli, R. Kohn and L. Nirenberg, Partial regularity of suitable weak solutions of the Navier-Stokes equations, Comm. on Pure and Appl. Math. 35 (1982), 771-831.

[3] M. Feistauer, Mathematical Methods in Fluid Dynamics, Pitman Monographs and Surveys in Pure and Applied Mathematics 67, Longman Scientific \& Technical, Harlow, 1993.

[4] G. P. Galdi, An introduction to the Navier-Stokes initial-boundary value problem, in: Fundamental Directions in Mathematical Fluid Mechanics, G. P. Galdi, J. Heywood, R. Rannacher (eds.), Adv. Math. Fluid Mech., Birkhäuser-Verlag, Basel, 2000, 1-98.

[5] E. Hopf, Über die Anfangswertaufgabe für die Hydrodynamischen Grundgleichungen, Math. Nachr. 4 (1951), 213-231.

[6] O. A. Ladyzhenskaya, The Mathematical Theory of Viscous Incompressible Flow, Gordon and Breach, New York 1969.

[7] J. Leray, Sur le mouvement d'un liquide visqueux emplissant l'espace, Acta Mathematica 63 (1934), 193-248.

[8] J. Nečas, Les Méthodes Directes en Théorie des Équations Elliptiques, Academia, Prague, 1967.

[9] J. Neustupa and P. Penel, The role of eigenvalues and eigenvectors of the symmetrized gradient of velocity in the theory of the Navier-Stokes equations, Comptes Rendus Acad. Sci. Paris Sér. I 336 (2003), 805-810.

[10] J. Neustupa and P. Penel, Regularity of a weak solution to the Navier-Stokes equation in dependence on eigenvalues and eigenvectors of the rate of deformation tensor, in: Trends in Partial Differential Equations of Mathematical Physics, J. F. Rodrigues, G. Seregin and J. M. Urbano (eds.), Birkhäuser-Verlag, Basel, 2004.

[11] R. Temam, Navier-Stokes Equations, North-Holland, Amsterdam, 1977. 